\title{
IMPROVEMENT OF A TAIL-PLANE STRUCTURAL MODEL USING VIBRATION TEST DATA
}

\author{
S. J. Guo \\ Department of Aerospace, Civil and Mechanical Engineering, \\ University of Hertfordshire, Hatfield, Hertfordshire, AL10 9AB, U.K.
}

\begin{abstract}
To achieve the best structural model improvement using vibration test data, main effort has been made to locate poor modelling regions as a guideline for subsequent model updating. The method presented and used in this paper is the energy error estimation method. In the method the difference between analytical and test data based energies at element scale is estimated to indicate any poor structural mass and stiffness modelling. As the result, poor modelling regions can be distinguished from those correctly modelled and the improvement of original structural model can be carried out effectively and accurately. To demonstrate the application of this method, a full-scale tail-plane structure has been studied by using simulated 'test' modes as a simulated case and using measured modes as a practical case. In both cases poor modelling regions of the original structural model have been accurately located. Subsequently, a significant improvement of the structural model with the reduction of average frequency error from original $2.2 \%$ down to $0.1 \%$ for the simulated case and from $4.6 \%$ to $1.8 \%$ for the practical case has been achieved.
\end{abstract}

Key Words: Tail-plane; Structural Model; Vibration Test;

\section{INTRODUCTION}

In the analysis of structural dynamics, accurate modal prediction is essential. For a practical structure of large size and complex configuration, however, modal errors due to simplification of structural modelling and poor estimation of structural parameters are almost inevitable. Poor analytical modelling may also occur if undetected damage (fault) exists in a structure causing overestimation of analytical stiffness in the local region. In order to improve the structural model efficiently, various methods based on available modal information from vibration test have been developed in the last two decades.

In the development of those methods, it has been noted that the major difficulty towards achieving the correct and unique solution is caused by the insufficiency (incompletion) of test modes. One type of the methods early developed to tackle this problem was proposed by Baruch [1], Berman and Nagy [2, 3], and was based on an optimal method, e.g. the Lagrange multiplier method. These methods have the advantage of simple and non-iterative model updating procedure and accurate matching of the predicted modes with measured modes. However these methods make the original system matrices reconstructed without preserving their physical meaning of element connection. Consequently the reconstructed model would not guarantee the modal correction beyond the range of measured modes.

The second type of developed methods includes the two-response method [4], the orthogonality and eigendata constraint methods [5], and the extended corrected modal 
constraint method [6]. Unlike the aforementioned first type of methods, only chosen matrix elements will be adjusted in these methods so that the original configuration of system matrices will be maintained. In practice however, the success of those methods heavily depend upon the accuracy and number of available measured modes. That is a crucial limit of the methods when applied to practical problems. One of the reasons for the above method limitation may be that both the location and magnitudes of the modelling errors in an analytical model need to be identified simultaneously during model updating. To avoid such difficulty, effort has been made to tackle the problem in two steps, i.e. the modelling error localisation followed by model correction. The error magnitudes of poorly modelled elements are not required at the first step of identifying their locations. Hence the conditions required for accurate result can be relaxed. By employing the sensitivity method for example, which may be classified as the third type of method, the sensitivity of eigendata errors [7] or orthogonality errors [8] to a small change of individual matrix element can be calculated. A non-zero sensitivity value indicates that the elements located in corresponding region may need improvement for accurate modal prediction. In practice however, not all modelling errors would be necessarily indicated by non-zero sensitivity values. This is mainly because the limited number of available measured modes may be insensitive to some of the modelling errors. On the other hand, not all non-zero sensitivity values necessarily represent the actual modelling errors. This is mainly because some regions to which the response is sensitive to modelling errors are actually correctly modelled. Normally an iterative performance is required for the model updating to converge.

Because of the similarity of poor modelling localisation to structural damage localisation using modal test data, some methods may be suitable for both applications. In the early 1990s Hearn and Testa [9] used modal strain energy for damage detection in structures. Contribution in developing the method has also been presented by Lim and Kashangaki [10]. Later further effort has been made to locate the structural damage based on the ratio of change in the modal strain energy caused by the damage [11]. Although this type of method is effective and may be applied to locating poor modelling, its accuracy of result, especially in the elements adjacent to the damage region, needs further improvement. In order to overcome this drawback, an energy error estimation method has been proposed and applied to both model improvement [12] and damage localisation [13] by Guo and Hemingway. This method is based on estimating the analytical and modal test data based kinetic and strain energies at finite element scale. The differences in magnitude represent the existing energy errors and indicate poor mass and stiffness modelling or internal structural damage. In terms of modal strain energy calculation, this method is similar to the aforementioned methods. However, this current method also provides additional measure to distinguish poor modelling or damage regions from those correctly modelled. This makes difference and has the advantage over the previously developed similar methods. Further more from the estimated energy error, a correction factor for each element can be created and used to improve each individual element and hence the whole model.

In this present paper, effort has been made to apply the energy error estimation method to improve the model of a full-scale tail-plane structure. One simulated example using 'test' data and one practical case using measured modes have been demonstrated. In both cases poorly modelled regions of the original structural model have been located accurately. Subsequently, significant improvement of the structural model with reduction of average frequency error from $2.2 \%$ down to $0.1 \%$ for the simulated 'test' case and from $4.6 \%$ to $1.8 \%$ for the practical case has been achieved. 


\section{THE ENERGY ERROR ESTIMATION METHOD}

\subsection{MODELLING ERRORS INDICATED BY ENERGY ERRORS}

Using the finite element method, the stiffness and mass models for a structural system are normally represented in matrices $[K]_{\mathrm{a}}$ and $[M]_{\mathrm{a}}$ assembled from element models $\left[K_{i}\right]_{\mathrm{a}}$ and $\left[M_{i}\right]_{\mathrm{a}}$, i.e.

$$
[K]_{a}=\sum_{i=1}^{n}\left[K_{i}\right]_{a} \quad \text { and } \quad[M]_{a}=\sum_{i=1}^{n}\left[M_{i}\right]_{a}
$$

where $\mathrm{n}$ represents the total number of elements in the system.

In a design process, unacceptable difference may be found between the predicted and measured modes of a structural system. In such case, it is usual to suspect that errors arise from the structural models under the assumption of reliable measured modes. The correct yet unknown stiffness and mass models, $[K]_{\mathrm{c}}$ and $[M]_{\mathrm{c}}$, may be then represented in terms of the original stiffness and mass matrices and their error matrices, $\left[\Delta K_{i}\right]$ and $\left[\Delta M_{i}\right]$, as follows

$$
[K]_{c}=\sum_{i=1}^{n}\left(\left[K_{i}\right]_{a}+\left[\Delta K_{i}\right]\right) \quad \text { and } \quad[M]_{c}=\sum_{i=1}^{n}\left(\left[M_{i}\right]_{a}+\left[\Delta M_{i}\right]\right)
$$

The modelling errors would cause difference between the analytical and test-data based energies, which are defined here as the strain and kinetic energy errors, $\Delta S_{j}$ and $\Delta T_{j}$, of the system as represented below.

$$
\begin{gathered}
\Delta S_{j}=\frac{1}{2}\{\bar{\Phi}\}_{j a}^{T} \sum_{i=1}^{n}\left[K_{i}\right]_{a}\{\bar{\Phi}\}_{j a}-\frac{1}{2}\{\bar{\Phi}\}_{j t}^{T} \sum_{i=1}^{n}\left(\left[K_{i}\right]_{a}+\left[\Delta K_{i}\right]\right)\{\bar{\Phi}\}_{j t} \\
\Delta T_{j}=\frac{1}{2} \lambda_{j a}\{\bar{\Phi}\}_{j a}^{T} \sum_{i=1}^{n}\left[M_{i}\right]_{a}\{\bar{\Phi}\}_{j a}-\frac{1}{2} \lambda_{j t}\{\bar{\Phi}\}_{j t}^{T} \sum_{i=1}^{n}\left(\left[M_{i}\right]_{a}+\left[\Delta M_{i}\right]\right)\{\bar{\Phi}\}_{j t}
\end{gathered}
$$

where $\lambda_{\mathrm{ja}}$ and $\lambda_{\mathrm{jt}}$ are the jth analytical and measured eigenvalues, $\{\bar{\Phi}\}_{j a}$ and $\{\bar{\Phi}\}_{j t}$ the jth analytical and test-data based mass-normalised mode shape divided by $\sqrt{2 \lambda_{j a}}$ and $\sqrt{2 \lambda_{j t}}$ respectively. In the above equations, since each of the terms is scaled to unit value, both $\Delta S_{j}$ and $\Delta T_{j}$ would be zero if $\left[\Delta K_{i}\right]$ and $\left[\Delta M_{i}\right]$ were included. In the initial analysis stage however, $\left[\Delta K_{i}\right]$ and $\left[\Delta M_{i}\right]$ are unknown, hence $[K]_{C}$ and $[M]_{C}$ are normally replaced by $[K]_{a}$ and $[M]_{a}$. In such case, the system energy errors $\Delta S_{j}$ and $\Delta T_{j}$ in a particular mode can be approximated below and become non-zero.

$$
\Delta S_{j} \approx \frac{1}{2}\left(\{\bar{\Phi}\}_{j a}^{T} \sum_{i=1}^{n}\left[K_{i}\right]_{a}\{\bar{\Phi}\}_{j a}-\{\bar{\Phi}\}_{j t}^{T} \sum_{i=1}^{n}\left[K_{i}\right]_{a}\{\bar{\Phi}\}_{j t}\right) \approx\{\bar{\Phi}\}_{j t}^{T} \sum_{i=1}^{n}\left[K_{i}\right]_{a}\{\Delta \bar{\Phi}\}_{j}
$$




$$
\begin{aligned}
\Delta T_{j} \approx \frac{1}{2}\left(\lambda_{j a}\{\bar{\Phi}\}_{j a}^{T} \sum_{i=1}^{n}\left[M_{i}\right]_{a}\{\bar{\Phi}\}_{j a}-\lambda_{j t}\{\bar{\Phi}\}_{j t}^{T} \sum_{i=1}^{n}\left[M_{i}\right]_{a}\{\bar{\Phi}\}_{j t}\right) \\
\approx \lambda_{j t}\{\bar{\Phi}\}_{j t}^{T} \sum_{i=1}^{n}\left[M_{i}\right]_{a}\{\Delta \bar{\Phi}\}_{j}+\Delta \lambda_{j}\{\bar{\Phi}\}_{j t}^{T} \sum_{i=1}^{n}\left[M_{i}\right]_{a}\{\bar{\Phi}\}_{j t}
\end{aligned}
$$

where $\Delta \lambda_{\mathrm{j}}=\lambda_{\mathrm{ja}}-\lambda_{\mathrm{jt}}$ and $\{\Delta \bar{\Phi}\}_{j}=\{\bar{\Phi}\}_{j a}-\{\bar{\Phi}\}_{j t}$ represent the frequency and modal errors of the jth mode respectively.

It is assumed here that a spatially complete set of measurement is available. In practice, analytical values will be employed to make the spatially incomplete measured modes expanded and completed. Equation (4) shows that the system energy errors $\Delta S_{j}$ and $\Delta T_{j}$ are the sum of element energy errors $\Delta S_{i j}$ and $\Delta T_{i j}$ in a particular mode, which are represented below.

$$
\begin{gathered}
\Delta S_{i j} \approx\{\bar{\Phi}\}_{j t}^{T}\left[K_{i}\right]_{a}\{\Delta \bar{\Phi}\}_{j} \\
\Delta T_{i j} \approx \lambda_{j t}\{\bar{\Phi}\}_{j t}^{T}\left[M_{i}\right]_{a}\{\Delta \bar{\Phi}\}_{j}+\Delta \lambda_{j}\{\bar{\Phi}\}_{j t}^{T}\left[M_{i}\right]_{a}\{\bar{\Phi}\}_{j t}
\end{gathered}
$$

For those correctly modelled elements, $\left[\Delta \mathrm{K}_{\mathrm{i}}\right]$ and $\left[\Delta \mathrm{M}_{\mathrm{i}}\right]$ don't exist and hence equation (5) gives accurate representation of their energy errors. Those energy errors may not be zero but small values due to modal error caused by poorly modelled elements elsewhere. Oppositely, large energy error values normally arise from those poorly modelled elements associated with $\left[\Delta K_{i}\right]$ and $\left[\Delta M_{i}\right]$. As more test modes are available and used, these element energy error values will be further increased and their difference from the correctly modelled elements will be further enlarged. The element energy errors in m number of test modes are represented as follows:

$$
\begin{gathered}
\Delta S_{i}=\sum_{j=1}^{m}\{\bar{\Phi}\}_{j t}^{T}\left[K_{i}\right]_{a}\{\Delta \bar{\Phi}\}_{j} \\
\Delta T_{i}=\sum_{j=1}^{m}\left(\lambda_{j t}\{\bar{\Phi}\}_{j t}^{T}\left[M_{i}\right]_{a}\{\Delta \bar{\Phi}\}_{j}+\Delta \lambda_{j}\{\bar{\Phi}\}_{j t}^{T}\left[M_{i}\right]_{a}\{\bar{\Phi}\}_{j t}\right)
\end{gathered}
$$

Energy errors are thus used to indicate the possibility, or degree of, element modelling errors.

\subsection{LOCALIZATION OF POORLY MODELLED ELEMENTS}

\subsubsection{Initial Localization}

According to equations (5) and (6), the total strain and kinetic energy errors of a system modelled by using ' $n$ ' number of elements can be estimated by using ' $m$ ' number of modes as follows:

$$
\Delta S=\sum_{i=1}^{n} \sum_{j=1}^{m} \Delta S_{i j} \quad \text { and } \quad \Delta T=\sum_{i=1}^{n} \sum_{j=1}^{m} \Delta T_{i j}
$$

A ratio of element against system energy errors is then recommended to evaluate stiffness and mass modelling errors for each of the elements in percentage 


$$
R K_{i}=\left(\Delta S_{i} /|\Delta S|\right) \cdot 100(\%) \quad \text { and } \quad R M_{i}=\left(\Delta T_{i} /|\Delta T|\right) \cdot 100 \quad(\%)
$$

The $R K_{i}$ and $R M_{i}$, which are used as an indicator of locating poor modelling, would have significantly higher magnitude for a poorly modelled element than correct one. Their sign also indicates an underestimation $(+)$ or overestimation $(-)$ of the stiffness or/and mass in the analytical model.

Although the above $R K_{i}$ and $R M_{i}$, provide a useful indicator to localise poorly modelled elements, the result is approximate and an uncertainty remains as weather a small value of $R K_{i}$ and $R M_{i}$, indicates a small modelling error or just accuracy error? Such uncertainty is especially concerned in the region adjacent to the poorly modelled elements. This problem occurring in the initial stage of modelling error localization causes difficulty to achieve high accuracy and reliability of locating and improving poor modelling. In order to solve the problem, further investigation has been carried out and a method to further distinguish the poorly modelled from correctly modelled elements has been proposed and described below.

\subsubsection{Final Localisation}

The method for further locating poorly modelled elements is based on the sign rather than the magnitude difference between the energy errors of poorly and correctly modelled elements. From equations (5-6), it is noted that the modal error $\{\Delta \Phi\}_{\text {j }}$ plays major role in energy error estimation. This is because it contains more explicit and useful information about the location and magnitude of modelling errors than $\Delta \lambda_{\mathrm{j}}$. Since $\{\Delta \Phi\}_{\mathrm{j}}$ in a poorly modelled region is caused directly by a local modelling error, it should remain the same sign for any mode. While in a correctly modelled region, where $\{\Delta \Phi\}_{\mathrm{j}}$ is caused indirectly by modelling errors beyond the region, the sign of $\{\Delta \Phi\}_{\mathrm{j}}$ and hence $\Delta S_{i j}$ and $\Delta T_{i j}$ may vary in different mode. Such sign variation can be worked out from equation (5) and used as an indicator to distinguish the correctly modelled elements from those poorly modelled ones. For example, if the sign of $\Delta S_{i j}$ and/or $\Delta T_{i j}$ for the ith element changes when using a different mode, this element stiffness and/or mass may be identified as correctly modelled. The element thus can be excluded from the poor modelling region regardless of its initial $R K_{i}$ and $R M_{i}$ values. If the sign remains the same as mode varies, the element would be identified as poorly modelled and thus should be included in the poor modelling region for subsequent model improvement. Although it has been noted that some modes may play more significant role than others in modelling error detection, generally speaking the more measured modes are available and used, the more reliable result would be expected. In practice however, because of the inevitable measurement errors and incomplete measurements in both spatial and modal coordinates, this method may not guarantee to filter off all correctly modelled elements from the initially located poorly modelled regions. Nevertheless this method provides a useful tool to enhance the accuracy and reliability of the localization result. It is simple, efficient and accurate provided that the effect on $\{\Delta \Phi\}_{\mathrm{j}}$ by measurement errors is less than that by modelling errors.

\subsection{IMPROVEMENT OF POOR ELEMENT MODELLING}

After localizing the poorly modelled elements, model improvement may be carried out with confidence by evaluating the stiffness and/or mass error matrix $\left[\Delta \mathrm{K}_{\mathrm{i}}\right]$ and $\left[\Delta \mathrm{M}_{\mathrm{i}}\right]$, 
and then modifying the original $\left[\mathrm{K}_{\mathrm{i}}\right]_{\mathrm{a}}$ and/or $\left[\mathrm{M}_{\mathrm{i}}\right]_{\mathrm{a}}$. Assuming $\left[\Delta \mathrm{K}_{\mathrm{i}}\right]$ and $\left[\Delta \mathrm{M}_{\mathrm{i}}\right]$ are a fraction of $\left[\mathrm{K}_{\mathrm{i}}\right]_{\mathrm{a}}$ and $\left[\mathrm{M}_{\mathrm{i}}\right]_{\mathrm{a}}$ of each element respectively, an approximation of them may be obtained by using the estimated $R K_{i}$ and $R M_{i}$ as follows:

$$
\left[\Delta \mathrm{K}_{\mathrm{i}}\right]=F K_{i}\left[\mathrm{~K}_{\mathrm{i}}\right]_{\mathrm{a}} \quad \text { and } \quad\left[\Delta \mathrm{M}_{\mathrm{i}}\right]=F M_{i}\left[\mathrm{M}_{\mathrm{i}}\right]_{\mathrm{a}}
$$

where $F K_{i}=F_{k} \times R K_{i}$ and $F M_{i}=F_{m} \times R M_{i}$ with $F_{k}$ and $F_{m}$ representing weighting factors for stiffness and mass modelling improvement respectively.

An initial model improvement can be achieved by substituting the above estimated $\left[\Delta \mathrm{K}_{\mathrm{i}}\right]$ and $\left[\Delta \mathrm{M}_{\mathrm{i}}\right]$ back into equation (2). Based on the improved model, further improvement can be carried out by repeating the above procedure. As the model is further improved, the remaining $\left[\Delta \mathrm{K}_{\mathrm{i}}\right]$ and $\left[\Delta \mathrm{M}_{\mathrm{i}}\right]$ hence $R K_{i}$ and $R M_{i}$ would become smaller. Such iterative implement can be carried out until the estimated $R K_{i}$ and $R M_{i}$ converge to a specified small value. Consequently the accuracy of predicted modes based on the improved model would be increased to achieve a minimum difference from the measured modes. Such mode difference is assessed here by an average frequency error $\Delta \mathrm{f}$ and mode shape error $\Delta \Phi$ represented as follows.

$$
\Delta f=\frac{1}{m} \sum_{j=1}^{m} \frac{\left|f_{a j}-f_{t j}\right|}{f_{t j}} \cdot 100 \quad(\%) \quad \text { and } \quad \Delta \Phi=\frac{1}{m} \sum_{j=1}^{m}\left(\frac{1}{n} \sum_{i=1}^{n} \frac{\left|\left(\Delta \Phi_{i}\right)_{j}\right|}{\left(\Phi_{i}\right)_{t j}}\right) \cdot 100
$$

\section{APPLICATION TO A TAIL-PLANE STRUCTURE}

A full-scale tail-plane structure of $5.95 \mathrm{~m}$ in span and $1.83 \mathrm{~m}$ in mainframe length as shown in Figure 1 was used for demonstration. The tail-plane is suspended by an elastic bungee at the front end of its main frame and seated on two vertical beams at its back ends. In the current demonstration of model improvement, the tail-plane structural modelling was largely simplified by using 3-D beam elements as illustrated in Figure 2.

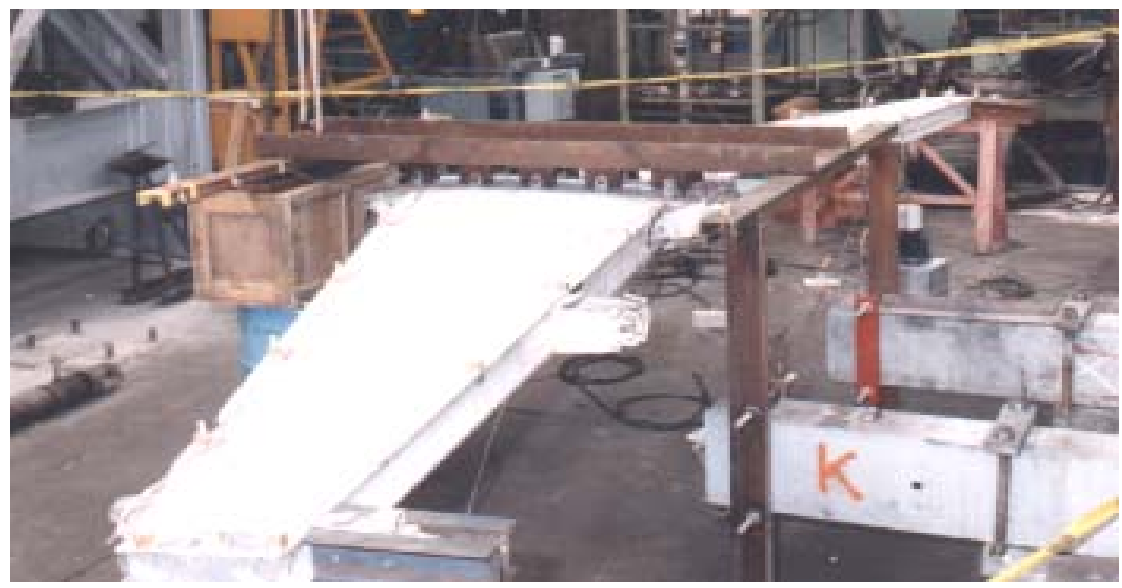

Figure 1. A view of the tail-plane set-up for vibration test

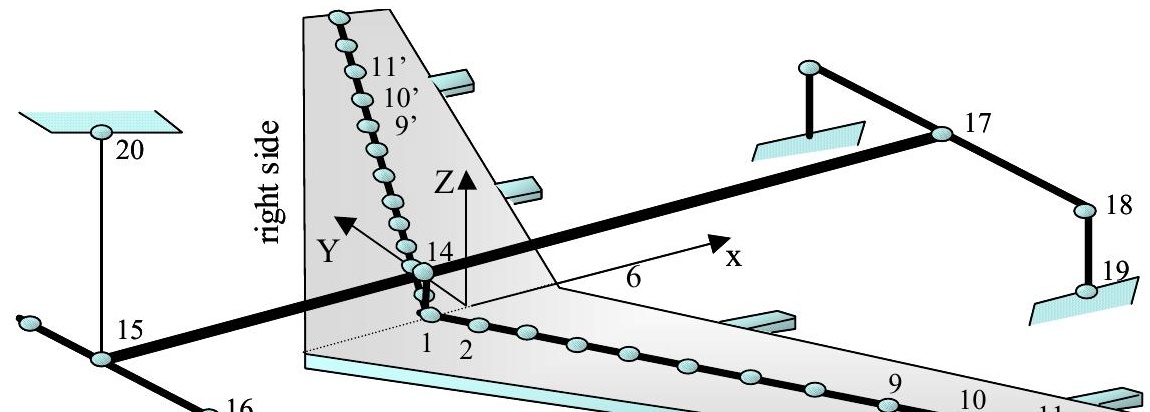


Figure 2. The analytical beam model of the tail-plane structure

The structure was represented by a stiffness matrix of $192 \times 192$ order assembled from each of the beam elements having $12 \times 12$ order stiffness matrix. Similarly, a mass matrix of the same order assembled from nodal mass matrix of $6 \times 6$ order was created to represent the mass properties of the structure. Considering the symmetric configuration of the structure, only half of the structure was modelled. The modal analysis for symmetric and anti-symmetric modes was carried out separately. The first 9 modes with frequency values listed in Table 1 were obtained as the analytical results.

Table 1. Predicted frequencies $(\mathrm{Hz})$ of the tail-plane analytical model

\begin{tabular}{c|ccccccccc}
\hline Mode No. & 1 & 2 & 3 & 4 & 5 & 6 & 7 & 8 & 9 \\
\hline Symmetric & $1.77^{*}$ & $9.83^{*}$ & 11.83 & 24.03 & 41.43 & 42.13 & 60.10 & 68.31 & 93.00 \\
Anti-symmetric & $4.77^{*}$ & 5.39 & 11.66 & 26.67 & 43.33 & 58.48 & 61.49 & 72.08 & 89.59 \\
\hline$*$
\end{tabular}

\subsection{MODEL IMPROVEMENT USING SIMULATED ‘TEST’ MODES (CASE 1)}

This first example is aimed to demonstrate the analysis procedure of the method. To obtain a set of simulated modes as our 'test' data, a 'test' structural model was created. Taking the same configuration and beam modelling as the analytical model shown in Figure 2, the 'test' structural model has $10 \%$ more mass in element 11 , and $10 \%$ less bending and torsion rigidity in elements $9 \& 10$. To keep the configuration symmetrical, the same difference as above was made on the opposite (right) side of the 'test' model. In this example, although eight symmetrical modes of the 'test' structure have been predicted as shown in Table 1, only the six flexible modes, i.e. modes $3-8$ were used as available 'test' modes in the model improvement procedure as described below. In this idealized example, the 'test' modes are assumed to be spatially complete and noise-free. Hence excellent modelling error locating result is expected.

\subsubsection{Modelling error localization}

Using the analytical model and the six 'test' modes in equations (6-8), an initial energy error distribution of the analytical model was obtained as shown in Figure 3 . The significantly large values of $\mathrm{RK}_{\mathrm{i}}$ and $\mathrm{RM}_{\mathrm{i}}$ indicate that poor stiffness modelling is most likely to occur in elements 9 and 10, and poor mass modelling in element 11. Due to symmetric configuration, similar modelling errors are also expected on the opposite side of the analytical model. It is noted that non-zero $\mathrm{RK}_{\mathrm{i}}$ and $\mathrm{RM}_{\mathrm{i}}$ values also appear beyond those elements. However they could be caused by approximation of the method or very small modelling errors since their values are significantly small. 
In order to further distinguish the poorly modelled elements from the correctly modelled ones, effort has been made to clear the above diagram of initial modelling error distribution. Using $\mathrm{Eq}(5)$ in this second stage, the sign of energy error $\Delta \mathrm{S}_{\mathrm{ij}}$ and $\Delta \mathrm{T}_{\mathrm{ij}}$ for each element and mode was obtained. Figure 4 shows that for all elements apart from $9 \&$ 10 , the sign of $\Delta \mathrm{S}_{\mathrm{ij}}$ changes at least once when a different mode is used. This reinsures us that only the stiffness of elements $9 \& 10$ is poorly modelled. Similarly, Figure 5 shows that only elements $6 \& 11$ contain mass modelling errors, while the other possible errors identified initially can be cleared away from Figure 3. As the result, a much clearer view of modelling error distribution can be obtained as shown in Figure 6. Comparing with the analytical model, the negative $\mathrm{RK}_{9}$ and $\mathrm{RK}_{10}$ indicate that the 'test' structure actually has smaller stiffness within its elements 9 and 10 region. While the positive $\mathrm{RM}_{\mathrm{i}}$ indicates that more mass actually exists in the region of element 11 .

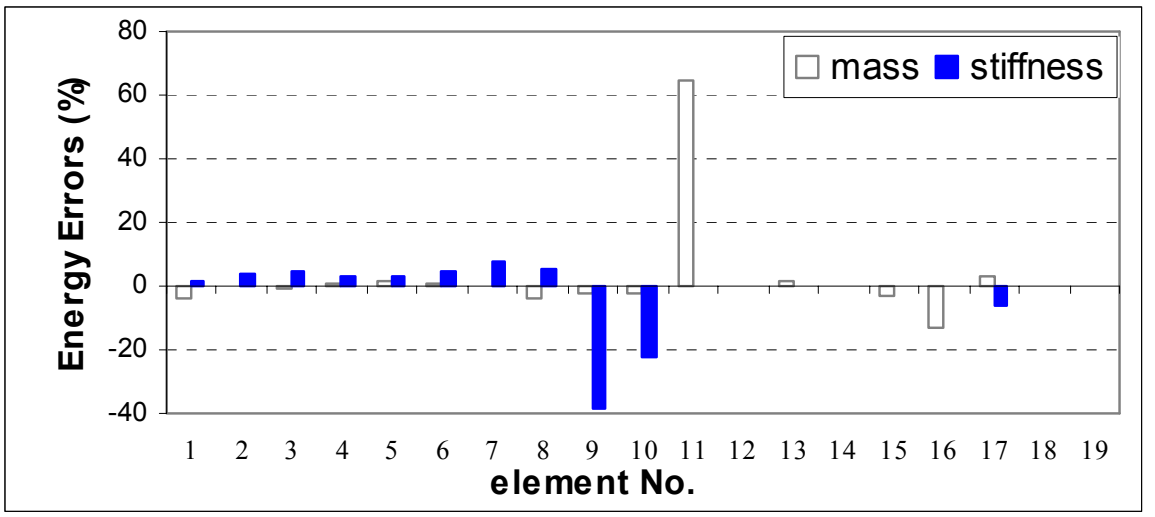

Figure 3. Energy Errors Indicating the Modelling Error Distribution

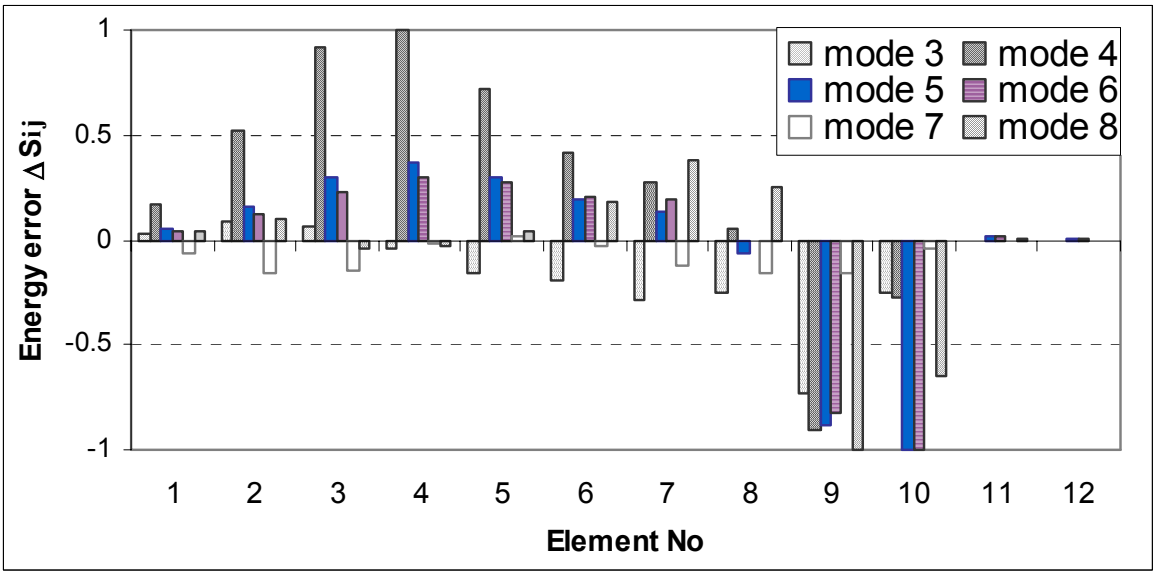

Figure $4 \mathrm{a}$. The sign variation of $\Delta \mathrm{S}_{\mathrm{ij}}$ along the tail-plane

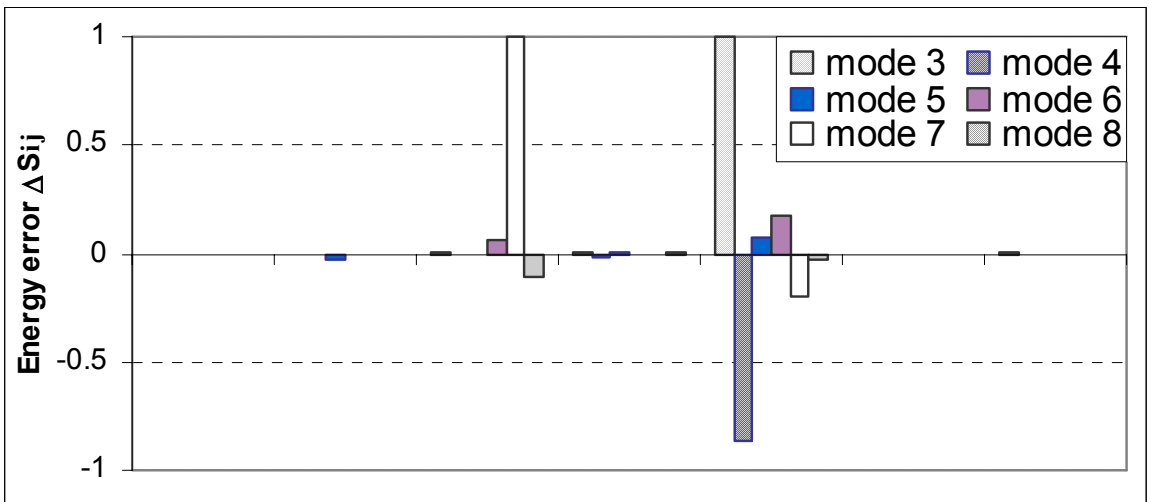


Figure $4 \mathrm{~b}$. The sign variation of $\Delta \mathrm{S}_{\mathrm{ij}}$ along the supporting beams

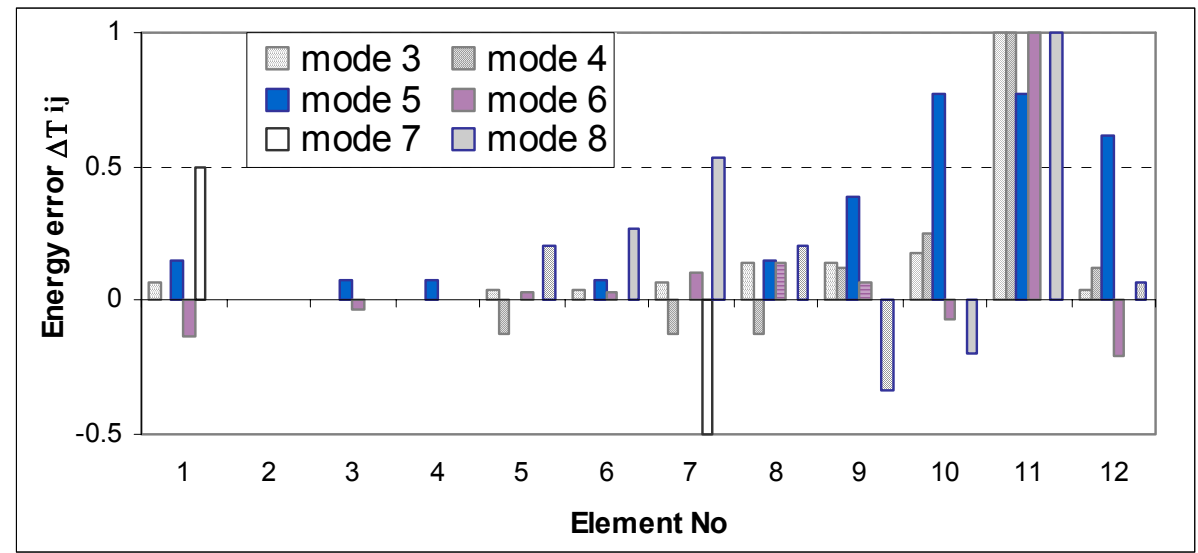

Figure 5a. The sign variation of $\Delta \mathrm{T}_{\mathrm{ij}}$ along the tail-plane

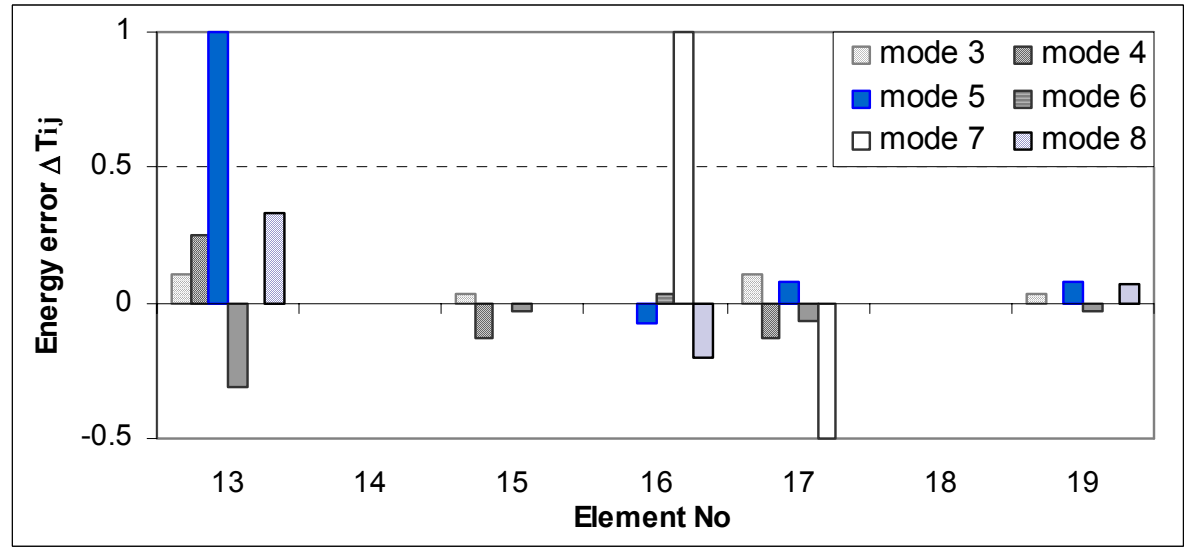

Figure $5 \mathrm{~b}$. The sign variation of $\Delta \mathrm{T}_{\mathrm{ij}}$ along the supporting beams

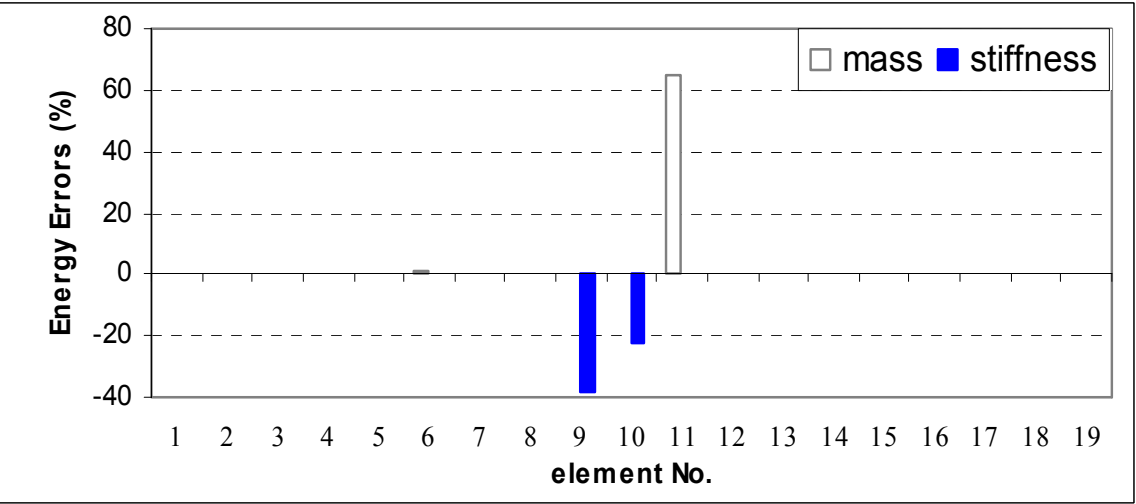


Figure 6. A clear view of modelling error localization

\subsubsection{Model Improvement}

Having localized the modelling errors as shown in Figure 6, the subsequent model improvement may be carried out by using equations (2), (8) \& (9) in an iterative manner or minimising $\Delta \mathrm{f}$ in a trial and error approach. In this example, the above estimated $\mathrm{RK}_{\mathrm{i}}, \mathrm{RM}_{\mathrm{i}}$ and equation (9) with weighting factors $F_{k}=0.4$ and $F_{m}=0.09$ were used to evaluate $\left[\Delta \mathrm{K}_{\mathrm{i}}\right.$ ] and $\left[\Delta \mathrm{M}_{\mathrm{i}}\right]$, which were then substituted into equation (2) to improve the original $[\mathrm{K}]_{\mathrm{a}}$ and $[\mathrm{M}]_{\mathrm{a}}$.

Since the model could not be completely corrected in one performance as above, the same procedure were repeated based on the previously improved model until the remaining errors became smaller than a specified value. In this example, the iteration was carried out until the evaluated $F_{k} \times \mathrm{RK}_{\mathrm{i}}$ and $F_{m} \times \mathrm{RM}_{\mathrm{i}}$ became less than $3 \%$ of their first iteration values as shown in Table 2 . In the model improvement, $F_{m} \times \mathrm{RM}_{6}$ for element 6 was too small and hence ignored. The sum of $F_{k} \times \mathrm{RK}_{\mathrm{i}}$ and $F_{m} \times \mathrm{RM}_{\mathrm{i}}$ from above iterations gives a ratio of the final evaluated $\left[\Delta \mathrm{K}_{\mathrm{i}}\right]$ against the original $[\mathrm{K}]_{\mathrm{a}}$ and $\left[\Delta \mathrm{M}_{\mathrm{i}}\right]$ against $[\mathrm{M}]_{\mathrm{a}}$ respectively. According to equations (2) \& (9), the result shows that the original stiffness for elements $9 \& 10$ should be reduced by $11 \%$ and the original mass in element 11 should be increased by $9.2 \%$ as final improvement. As a result, the modelling errors in the analytical model have been reduced from initial $10 \%$ down to less than $1 \%$.

Table 2. Evaluated factors for model improvement during iteration

\begin{tabular}{c|c|c|c|c|c}
\hline \multicolumn{1}{c|}{ Iteration No. } & 1 & 2 & 3 & 4 & \\
\hline$F_{k} \times \mathrm{RK}_{9}$ for element 9 & -0.13 & 0.02 & -0.0036 & & $\sum=-0.11$ \\
\hline$F_{k} \times \mathrm{RK}_{10}$ for element 10 & -0.09 & -0.02 & 0.0002 & & $\sum=-0.11$ \\
\hline$F_{m} \times \mathrm{RM}_{11}$ for element 11 & 0.056 & 0.027 & 0.007 & 0.002 & $\sum=0.092$ \\
\hline
\end{tabular}

Comparing with the 'measured' modes shown in Table 3, mode errors of the improved analytical model have been largely reduced. For example, the average mode error for modes $3-8$ has been reduced from $2.23 \%$ to $0.09 \%$.

Table 3. Comparison between analytical and 'test' frequencies $(\mathrm{Hz})$

\begin{tabular}{c|c|c|c|c|c|c|c|c}
\hline Mode No. & $1^{*}$ & $2^{*}$ & 3 & 4 & 5 & 6 & 7 & 8 \\
\hline original $\mathrm{f}_{\mathrm{a}}$ & 1.77 & 9.83 & 11.83 & 24.03 & 41.43 & 42.13 & 60.10 & 68.31 \\
\hline error $\Delta \mathrm{f}(\%)$ & 0.91 & 0.60 & 2.87 & 0.54 & 3.45 & 3.74 & 0.10 & 2.66 \\
\hline improved $\mathrm{f}_{\mathrm{a}}$ & 1.76 & 9.77 & 11.52 & 23.90 & 40.09 & 40.68 & 60.04 & 66.48 \\
\hline error $\Delta \mathrm{f}(\%)$ & 0.0 & 0.02 & 0.17 & 0.00 & 0.10 & 0.17 & 0.00 & 0.09 \\
\hline 'test' $\mathrm{f}_{\mathrm{t}}$ & 1.76 & 9.77 & 11.50 & 23.90 & 40.05 & 40.61 & 60.04 & 66.54 \\
\hline \multicolumn{7}{c}{ rigid-body mode }
\end{tabular}




\subsection{MODEL IMPROVEMENT USING MEASURED MODES (CASE 2)}

In this second example, improvement of the original analytical model shown in Figure 2 was carried out by using only four measured modes from a vibration test of the tail-plane shown in Figure 1. In the vibration test, most of the 29 accelerometers were mounted along the front and rear spars of the tail-plane as shown in Figure 7 and limited to measuring the vertical movement. By applying the quadratic interpolation method, the out of plane measurements were used to obtain the intermediate unmeasured displacements, i.e. the out of plane vertical and torsional displacements and span-wise bending slopes. Due to the lack of in plane measurements however, analytical values were used as the displacements at the intermediate unmeasured DOFs, i.e. in plane translations and yaw. However, it should be noted that these analytical values do not contribute to any energy errors. Hence some compromise in the performance of the error localisation and accuracy of the model improvement can be expected.

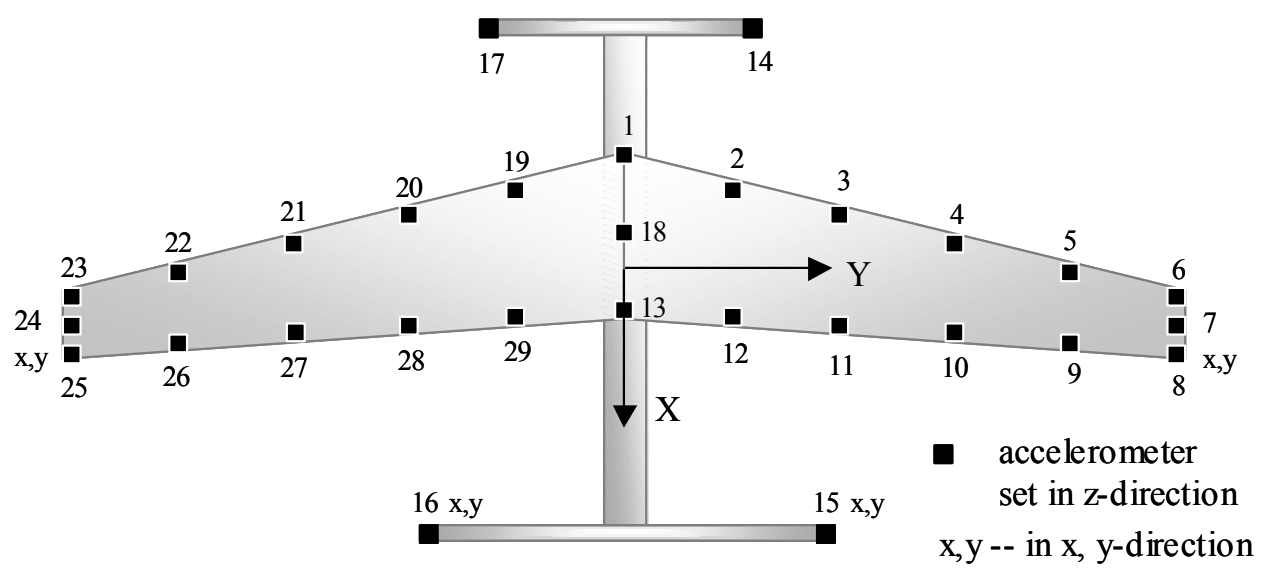

Figure 7. Top view of the measurement points on the tail-plane

Table 4. Measured frequencies $(\mathrm{Hz})$ from the tail-plane vibration test

\begin{tabular}{c|ccccccccc}
\hline Mode No. & 1 & 2 & 3 & 4 & 5 & 6 & 7 & 8 & 9 \\
\hline Symmetric & $1.76^{*}$ & $9.64^{*}$ & 13.02 & 23.71 & -- & -- & 59.35 & 65.86 & -- \\
Anti-symmetric & $5.19 *$ & 5.39 & -- & 25.73 & 44.48 & -- & -- & -- & 81.33 \\
\hline
\end{tabular}

\subsubsection{Modelling error localization}

In this example, considering the symmetric vibration case and half of the analytical model, only the four measured symmetric modes $3,4,7 \& 8$ were used in the analysis. The rest of modes are listed only for comparison. By applying the EEE method, the total strain and kinetic energy errors on both sides of the analytical model were estimated from equations (6) \& (7) and listed in Table 5. It shows that the strain energy error $\mathrm{RK}_{\mathrm{i}}$ on the left side of the model is obviously larger than that on the right side. Hence large stiffness modelling errors is likely to exist on the left side of the analytical model. It also shows that the kinetic energy error $\mathrm{RM}_{\mathrm{i}}$ is much lower than the $\mathrm{RK}_{\mathrm{i}}$ on both sides of the model. This 
indicates that the overall mass modelling errors in the analytical model is very small comparing with the stiffness errors.

Table 5. Total energy errors on both sides of the analytical model

\begin{tabular}{c|c|c}
\hline Total energy errors & Left side & Right side \\
\hline Strain $\Delta \mathrm{S}$ & 13.30 & 3.60 \\
Kinetic $\Delta \mathrm{T}$ & 1.61 & 1.37 \\
\hline
\end{tabular}

To have a detailed view of the modelling error distribution, $\mathrm{RK}_{\mathrm{i}}$ and $\mathrm{RM}_{\mathrm{i}}$ in each element of the model were estimated by using equation (8) and the results were shown in Figure 8. It can be seen from Figure 8a that the stiffness errors are most likely to be located in the elements 9 and 10 on the left side, and also at the root elements of the analytical model. The negative sign of the errors indicate smaller stiffness in the actual structure than that being modelled. Figure $8 \mathrm{~b}$ shows an even distribution of $\mathrm{RM}_{\mathrm{i}}$ indicating mass modelling errors of the model. Since the maximum $\mathrm{RM}_{\mathrm{i}}$ value reaches only about $1.5 \%$ of the sum of $\mathrm{RM}_{\mathrm{i}}$ values, which is very small comparing with the $\mathrm{RK}_{\mathrm{i}}$ shown in Figure 8a, the mass modelling errors are ignored in the subsequent model improvement. Although the modelling error distributions obtained so far are reasonable clear, it would be desirable to have a clearer view for more accurate localization of the modelling errors, especially in the regions with relatively low $\mathrm{RK}_{\mathrm{i}}$ values. At this stage, equation (5) was used to get the sign variation of element energy error for each of the modes as shown in Figure 9 (elements beyond 13 are excluded due to their small errors as shown in Figure 8).

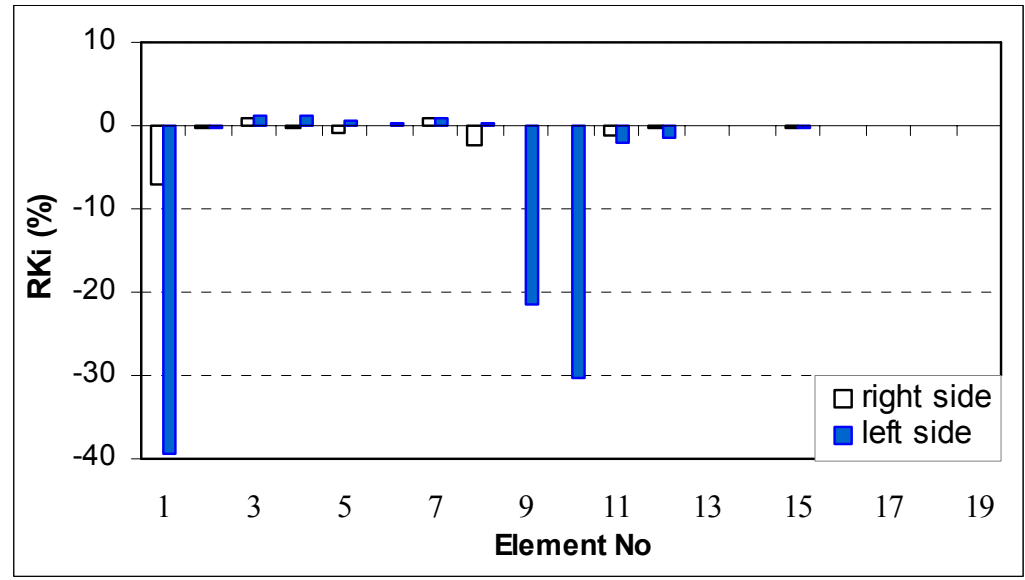

Figure 8a. Stiffness modelling error distribution indicted by $\mathrm{RK}_{\mathrm{i}}$

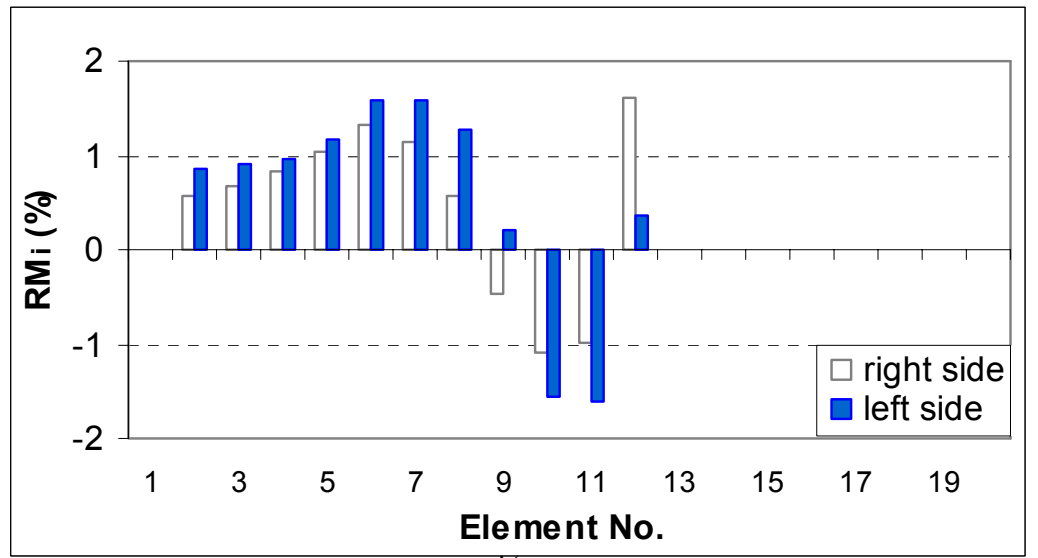


Figure $8 \mathrm{~b}$. Mass modelling error distribution indicated by $\mathrm{RM}_{\mathrm{i}}$

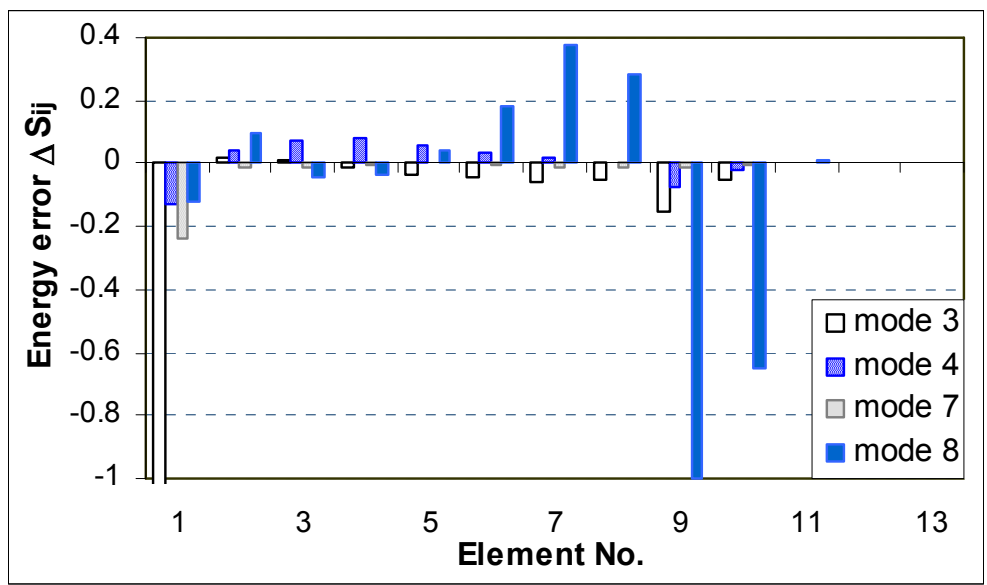

Figure 9a. Elemental strain energy error for each mode

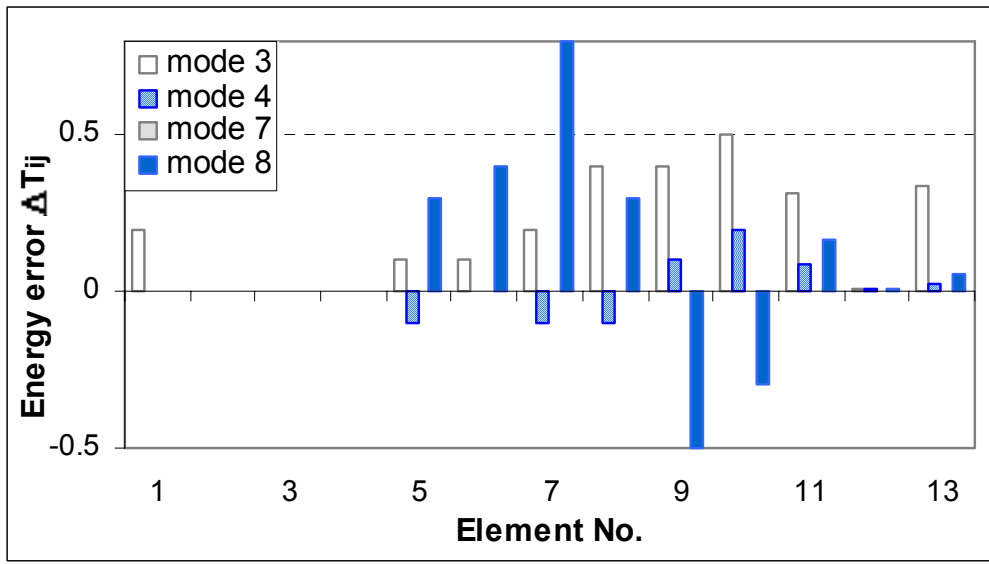

Figure 9b. Elemental kinetic energy error for each mode

Fig 9a shows that only the signs of strain energy errors for elements $1,9 \sim 12$ remain the same for all the modes. This indicates that only those elements were poorly modelled in terms of stiffness, and the rest of elements were correctly modelled. Figure $9 \mathrm{~b}$ shows that none of the kinetic energy errors remains the same sign for different modes. This reinsures that the original mass modelling is good enough and thus no improvement is required in this case. Focusing on the stiffness modelling errors and clearing out those fault errors initially identified as shown in Figure 8a, a clearer view of locations of stiffness modelling errors for the left side of the tail-plane was obtained as shown in Figure 10.

The negative values of $\mathrm{RK}_{\mathrm{i}}$ shown in Figure 10 indicate that some local regions of the tail-plane actually have smaller stiffness than that of the original analytical model. Having a close look at the local structure where elements $9 \& 10$ were positioned as shown in Figure 
11, the large stiffness modelling errors located in this region can be proved to be due to a local structural damage. The small modelling errors remaining in the elements $11 \& 12$ may be due to the effect of the neighbouring structural damage and approximation of the method. The large modelling error of element 1 was likely caused by the model simplification of the junction between tail-plane and its main supporting beam as shown in Figures. $1 \& 2$. Those modelling errors caused significant differences between the predicted modes from the original model and measured modes of the structure as shown in Table 6.

\subsubsection{Analytical Model Improvement}

3.2.2.1. First improvement In order to improve the original analytical model, attention was then focused on the poor modelling regions to evaluate the stiffness error matrix $\left[\Delta \mathrm{K}_{\mathrm{i}}\right]$ for elements 1,9 and 10 using equation (9). By substituting the obtained $\left[\Delta \mathrm{K}_{\mathrm{i}}\right]$ into equation (2), an improved $[\mathrm{K}]_{\mathrm{a}}$ of $20 \%$ stiffness (EI, GJ) reduction for element $1 \& 9$, and $22 \%$ reduction for element 10 was obtained. Consequently, the average mode errors of the improved analytical model were reduced, from the original $\Delta \mathrm{f}=4.6 \%$ to $3.8 \%$ and from $\Delta \Phi=14.3 \%$ to $13.3 \%$ as shown in Table 6 . After the above first improvement however, stiffness modelling errors still remained in the analytical model as shown in Figure 10.

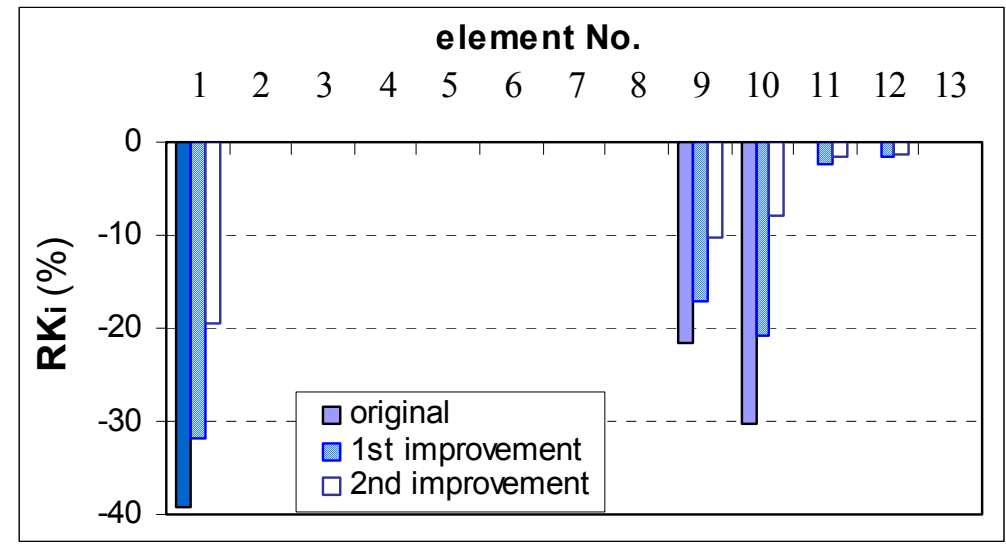

Figure 10. Improved localization of stiffness modelling errors

Table 6. Comparison between analytical and measured modes

\begin{tabular}{c|cccc|ccc|c}
\hline Mode & \multicolumn{4}{|c|}{ Symmetric Modes } & \multicolumn{2}{|c}{ Antisymmetric Modes } & Average \\
No. & 3 & 4 & 7 & 8 & 4 & 5 & 9 & mode error \\
\hline original f $(\mathrm{Hz})$ & 11.83 & 24.03 & 60.10 & 68.31 & 26.68 & 43.33 & 89.59 & $\begin{array}{c}\Delta \mathrm{f}=4.56 \% \\
\end{array}$ \\
\hline $\begin{array}{c}1^{\text {st }} \text { improved f } \\
\text { by EEE method }\end{array}$ & 11.74 & 23.96 & 60.02 & 65.86 & 26.45 & 43.29 & 88.82 & $\Delta \mathrm{f}=3.81 \%$ \\
\hline $\begin{array}{c}\text { 2nd improved f } \\
\text { by EEE method }\end{array}$ & 11.72 & 23.94 & 59.98 & 64.80 & 26.37 & 43.28 & 88.22 & $\Delta \mathrm{f}=3.90 \%$ \\
\hline Further improved & 13.30 & 23.80 & 60.20 & 66.00 & 25.80 & 43.20 & 85.60 & $\Delta \mathrm{f}=1.80 \%$ \\
\hline measured $\mathrm{f}_{\mathrm{t}}$ & 13.02 & 23.71 & 59.35 & 65.86 & 25.73 & 44.48 & 81.33 & \\
\hline
\end{tabular}




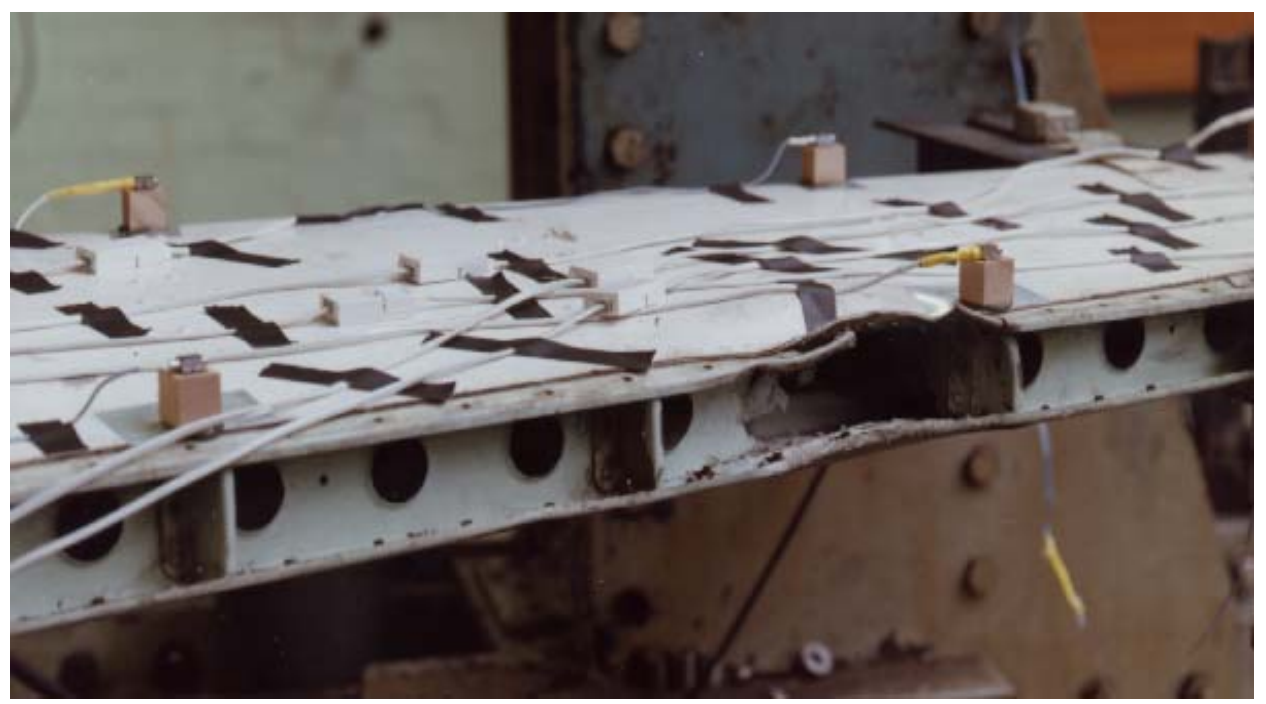

Figure 11. Structural damage on the left side of the tail-plane

3.2.2.2. Second improvement If the above procedure was repeated based on the improved model, a further $20 \%$ stiffness reduction for elements 1 \& 9 and $40 \%$ reduction for element 10 were estimated. Subsequently a further reduction of the remaining stiffness modelling errors was made after the second improvement as shown in Figure 10. However, comparing with the measured modes again as shown in Table 6, it was noted that the average $\Delta$ f was slightly increased from $3.81 \%$ up to $3.90 \%$ although the average $\Delta \Phi$ was reduced further from $13.25 \%$ to $10.15 \%$. It was also noted that such large reduction of stiffness would not give a good representation of the actual structure. It was then suspected that some modelling errors remained unidentified in the regions beyond those located so far. The failure to locate those errors may be mainly because of the lack of measurement along the supporting frames of the tail-plane. Hence, an alternative approach for further model improvement has to be considered.

3.2.2.3. Further improvement In this example, it was assumed that the measured frequencies were more accurate and reliable than mode shapes. Thus, an approach based on the analysis of frequency sensitivity to the variation of element stiffness parameters was used. In the analysis, only measured frequencies were used as the reference base to minimize the effect of inadequate measurement of mode shapes on further model improvement.

Firstly we focused on the region of junction between the tail-plane and its main supporting frame. The analytical model was updated by adding two more elements between nodes $1-15$ and $1-18$ as shown in Figure 12. This updated model should give a more realistic representation of the actual structure and provide more parameters for sensitivity analysis and model improvement in the region.

In the analysis, although all measured frequencies were considered, attention was paid mainly to the first symmetric mode and all the anti-symmetric modes, which had relatively large errors. It was found that the first symmetric mode was sensitive to the bending stiffness of the new elements $1-15,1-18$ and element $20-21$ at the back. The first anti-symmetric mode was also sensitive to the new elements $1-15,1-18$, but the third mode was more sensitive to elements $19-20 \& 20-21$. From the sensitivity analysis, bending \& torsion 
stiffness of the new elements 1-15, 1-18 was determined to be $16.53 \mathrm{~N} . \mathrm{m}^{2}$ and the bending stiffness of element $19-20$ was reduced from $6.612 \mathrm{~N} . \mathrm{m}^{2}$ to $4.959 \mathrm{~N} . \mathrm{m}^{2}$. Following the above stiffness updating, the mode errors were further reduced. A comparison between the frequencies of the original model, improved model using EEE method and further improved model using sensitivity method is shown in Table 6.

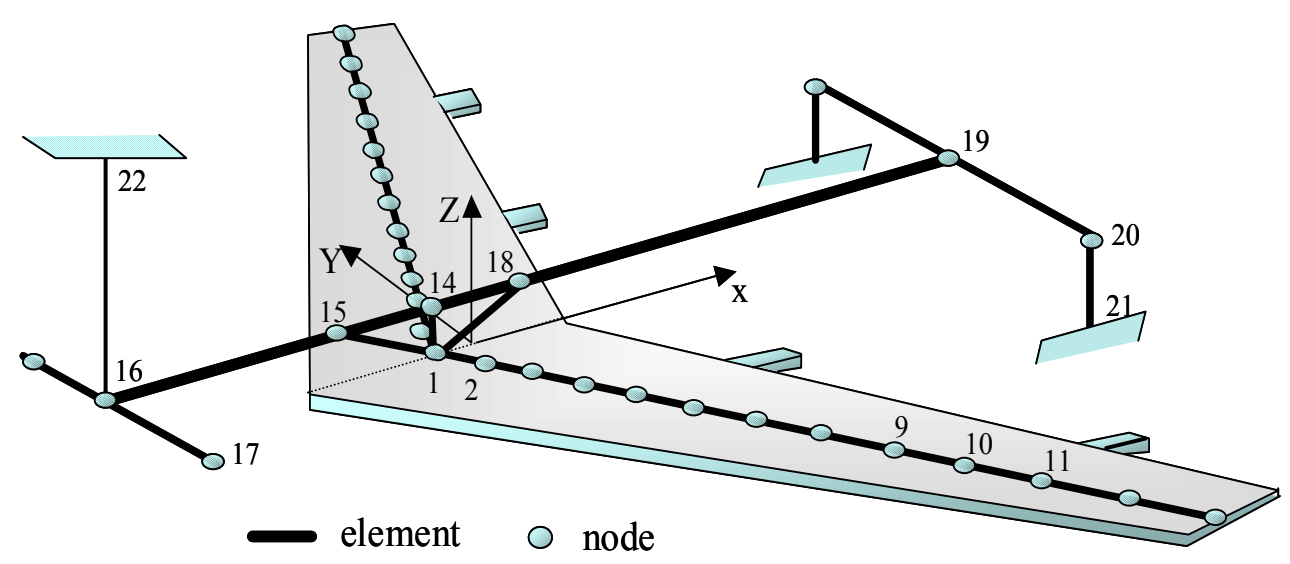

Figure 12. An updated analytical model for further improvement

\section{CONCLUDING REMARKS}

The energy error estimation method provides a feasible and efficient tool for model improvement based on a set of incomplete measured modes. The method is applicable to locating the regions of analytical modelling errors or structural damage. From the investigation of this current paper, the following conclusions can be extracted.

- By using this method, both the stiffness and mass modelling errors of an analytical model can be identified and localised.

- In the initial localisation of modelling errors, the result was approximate due to the effect of the limit of the method and available measured modes. However an additional measure proposed in this method can increase the localisation accuracy. This is the major advantage of the method in terms of accuracy.

- Although the method depends upon the quality of measured modes, the regions with significant modelling errors such as the damage region can be distinguished from good modelling regions.

- In the regions such as the supporting frames of the tail-plane where few measurements were made, the estimated energy errors won't be accurate enough to indicate modelling errors. In such case, sensitivity analysis based on measured frequencies provides an effective alternative.

- The model improvement is restricted to only those regions where modelling errors have been identified. This ensures the reliability and accuracy of the improved model.

- The improved analytical model retains its original matrix size and configuration and thus retains the physical description of the system.

Finally it has been noted in this method that some modes may play more significant role than others to locate modelling errors. This may raise a concern of mode sensitivity of the method. Generally speaking, the effect of mode on the accuracy of result would depend 
upon the structural configuration and modelling error locations. Although in practice we normally don't have much choice of the very limited available measured modes, an investigation into this modal sensitivity problem is recommended, but beyond the scope of this current paper.

\section{REFERENCES}

1. M. BARUCH 1978 AIAA Journal, Vol. 16, 1208 - 1210. Optimal procedure to correct of stiffness and flexibility matrices using vibration tests.

2. A. BERMAN 1979 AIAA Journal, Vol. 17, 1147 - 1148. Mass matrix correction using an incomplete set of measured modes.

3. A. BERMAN and E.J. NAGY 1983 AIAA Journal, Vol. 21, 1168 - 1173. Improvement of a large analytical model using test data.

4. S.R. IBRAHIM et al 1989 Proceedings of the $7^{\text {th }}$ IMAC, Las Vegas, $340-346$. A direct two-response approach for updating analytical dynamic models of structures with emphasis on uniqueness.

5. W.M. TO, R.M. LIN and D.J. EWINS 1990 Proceedings of the $8^{\text {th }}$ IMAC, $961-967$. A criterion for the localization of structural modification sites using modal data.

6. S.J. GUO $1993 \mathrm{PhD}$ thesis, University of Hertfordshire. Analytical dynamic model improvement using vibration test data

7. J.C. Chen, L.F. PERETTI and J.A. GARbA 1984 AIAA Paper, 84-1051, 478 - 489. Spacecraft structural system identification by modal test.

8. S.J. GuO and N.G. Hemingway 1991 Proceedings of the $9^{\text {th }}$ IMAC, $469-473$. Improvement of a FE model with dynamic reduction using test data.

9. G. HEARN and R.B. TESTA 1991 Journal of Structural Engineering ASCE 117, 30423063. Modal analysis for damage detection in structures.

10. T.W. LIM and T.A.L. KASHANGAKI 1994 AIAA Journal 32, 1049-1057. Structural damage detection of space truss structures using best achievable eigen-vectors.

11. Z.Y.SHI, S.S.LAW and L.M. ZHANG 1998 Journal of Sound \& Vibration 218(5), 825-844. Structural damage localization from modal strain energy change.

12. S.J. GuO and N.G. HemingwAY 1995 Journal of Mechanical Engineering Science (Part C) Vol. 209, 97-105. An energy error estimation method for improving analytical models using vibration modal test data.

13. S.J. GuO and N.G. HeMINGWAY 2000 Proceedings of Int. Conference on Advanced Problems in Vibration, 329-335. Structural damage localisation using energy error estimation method. 